\title{
A case of refractory seizure with cognitive impairment due to anti-GABA encephalitis
}

Adrian TH Hui *, MB BS, MRCP (UK), YO Lam, MB, BS, MRCP (UK), CK Chan, MB, BS, FHKAM (Medicine), KY Cheung, MB, ChB, FHKAM (Medicine), BH Fung, MB, BS, FHKAM (Medicine), PW Ng, MB, BS, FHKAM (Medicine)

Department of Medicine and Geriatrics, United Christian Hospital, Kwun Tong, Hong Kong

*Corresponding author: hth077@ha.org.hk

Hong Kong Med J 2016;22:509-11

DOI: $10.12809 / \mathrm{hkmj} 154604$

\section{Case report}

A 57-year-old man presented with first episode of loss of consciousness at work in February 2014. He experienced no symptoms prior to this syncope event. He was witnessed by his colleagues to have tonic rigidity over his upper limbs, with a bite mark evident over the lateral aspect of his tongue. $\mathrm{He}$ had experienced a right-sided temporal headache characterised as dull and persistent for 3 weeks prior to this incident without any precipitating factors or aura identified.

He enjoyed good past health and had been educated to high school level. He was currently working as an accountant. He did not smoke or drink, had no recent travel history, and was not on any long-term medication or taking herbs. He could not recall any febrile convulsion during childhood or significant family history of neurological diseases.

He was fully oriented and remained afebrile on admission. Routine blood tests (electrolytes, glucose, and inflammatory markers), physical examination, and initial computed tomographic (CT) brain were unremarkable. The patient refused lumbar puncture and was discharged against medical advice.

Three days after discharge, he was admitted to a private hospital with generalised tonic-clonic convulsion. Electroencephalography was normal and magnetic resonance imaging (MRI)/magnetic resonance angiography brain showed no mass lesion or infarct. He was discharged with a prescription of levetiracetam in view of the second episode of seizure.

He was again admitted to us in early March 2014 with breakthrough seizure and post-ictal drowsiness, despite good drug compliance and no identifiable precipitating factors. His seizure was initially controlled with levetiracetam with the addition of phenytoin, but he remained disoriented with confused speech. His Mini-Mental State Examination (MMSE) score was 14/30: his main deficit was delayed recall with failure to perform serial sevens and to copy a polygon. He developed a low-grade fever of $37.8^{\circ} \mathrm{C}$ with negative septic workup. Lumbar puncture did not reveal any evidence of central nervous system infection (white cell count, $<1 / \mathrm{mm}^{3}$, protein level not elevated, and negative culture result). Electroencephalography showed right temporal spikes only. He was prescribed empirical Augmentin (Sandoz, Australia) 1.2 g every 8 hours intravenously and his fever settled.

One week after admission, he experienced multiple episodes of generalised tonic-clonic seizure without progression to status epilepticus. Doses of both phenytoin and levetiracetam were increased along with the addition of valproic acid. His seizures abated but cognition was not improved. Electroencephalography was repeated and demonstrated left temporal polyspikes over F7/T3/T5 in addition to bitemporal sharp waves (Fig 1). Brain MRI was also repeated and showed no abnormality (Fig 2). Blood tests were unremarkable with normal electrolytes, negative tumour markers (alphafetoprotein/carcinoembryonic antigen/prostatespecific antigen), negative autoimmune markers (antinuclear antibodies/rheumatoid factor), normal thyroid function and lactate level, and human immunodeficiency virus-negative status. Lumbar puncture was repeated, and this time showed mild lymphocytic pleocytosis with white cell count of $9 / \mathrm{mm}^{3}$ (98\% lymphocyte count), protein level again not elevated, cerebrospinal fluid (CSF)serum glucose ratio of 0.53 (4.1/7.8). Both serum and CSF oligoclonal bands were present, and immunoglobulin $\mathrm{G}$ index was also elevated.

Both autoimmune encephalitis and post-ictal pleocytosis are important differential diagnoses to be considered. Serum and CSF were saved for the detection of auto-antibodies, although anti-Nmethyl D-aspartate (anti-NMDA), anti-voltagegated potassium channel (anti-VGKC), and antineuronal (anti-Hu/anti-Ri/anti-Yo) antibodies were all negative, anti-GABA ${ }_{B}$ antibodies were detected in both serum and CSF of this patient.

\section{Discussion}

Autoimmune encephalitis, previously labelled limbic encephalitis, was first described in the 1960s when patients with lung cancer also suffered from temporal lobe epilepsy, memory loss, and dementia features. ${ }^{1}$ It was once thought that limbic encephalitis 


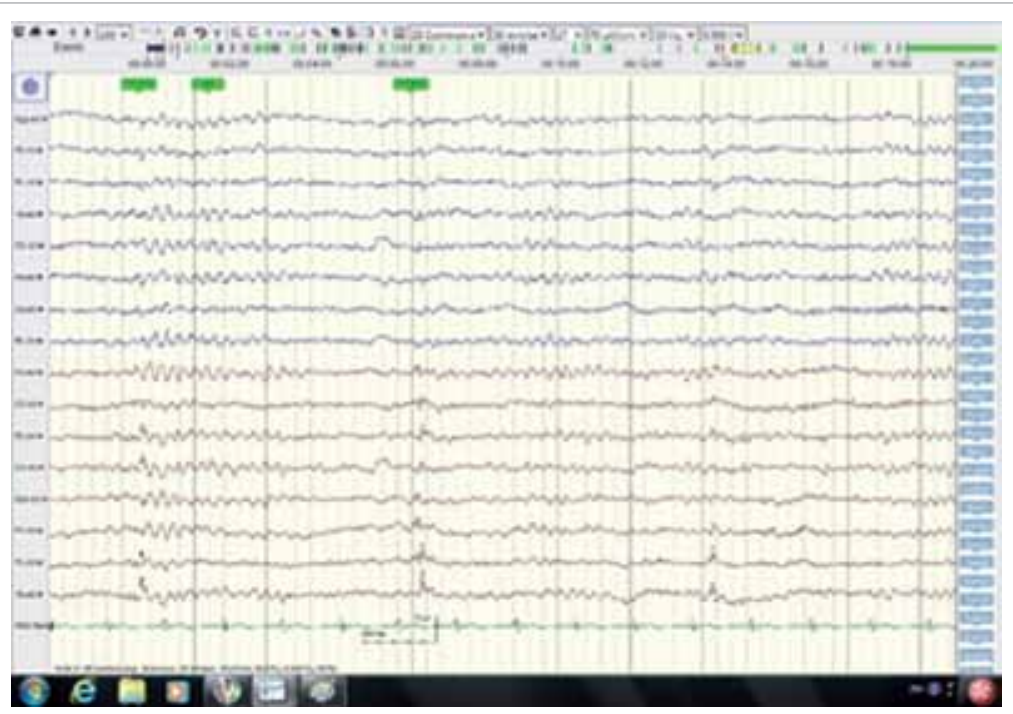

FIG I. Electroencephalography demonstrates left temporal polyspikes over F7/T3/ T5 in addition to bitemporal sharp waves

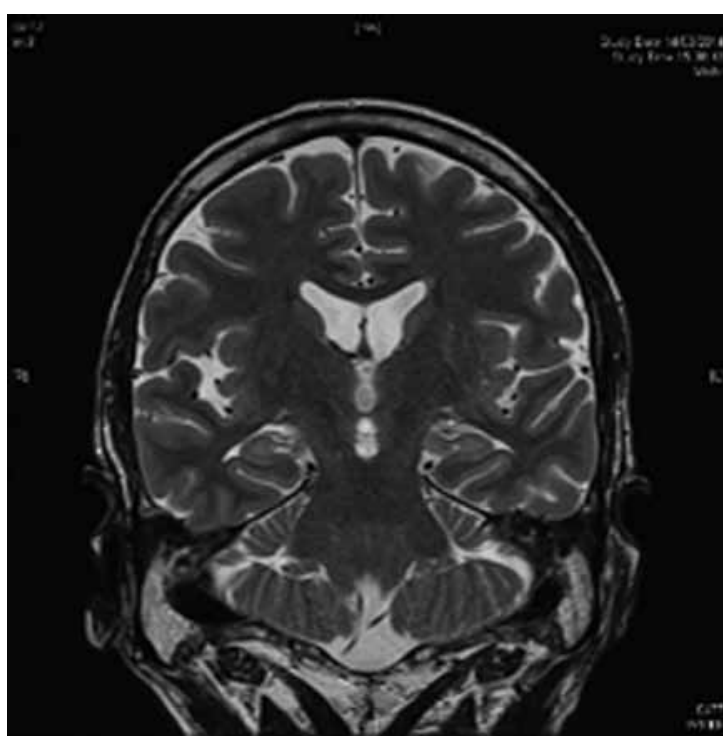

FIG 2. Magnetic resonance imaging of the brain showing no hyperintense mesio-temporal lobe signal change on T2 and fluid-attenuated inversion recovery

was always associated with malignancy. In 1985, the first onconeuronal antibody, anti-Hu antibody, was discovered in small-cell lung carcinoma patients. ${ }^{1}$ More antibodies were identified in subsequent years, namely CV2/CRMP5-Ab and Ma2-Ab, that target intracellular peptides being expressed on the cell membrane. ${ }^{1}$ At the turn of the 21 st century, patients who had features of limbic encephalitis did not necessarily develop malignancy, and those affected were usually young individuals who responded well to immunotherapy. Researchers later found antibodies in these patients that targeted cell membrane antigens that are receptors involved in synaptic transmission, plasticity, and neuronal excitability. The first such antibody discovered was VGKC. ${ }^{1}$

The pathophysiological mechanism involves both humeral and cellular immunity mediated by antibody production and cytotoxic $\mathrm{T}$ cells, respectively. ${ }^{1}$ The hippocampus and hypothalamus are most vulnerable during the active disease stage as the permeability of the blood-brain barrier is increased more than other regions of the brain, and is thereby more susceptible to autoimmune attack. ${ }^{2}$

Treatment modalities include removal of the underlying tumour and the antibodies. Thus far, no randomised controlled trials have analysed the efficacy of such treatment. Clinical experience would suggest that the first-line immunotherapy would be steroid as anti-inflammatory agent and intravenous immunoglobulin (IVIG)/plasma exchange for antibody removal. ${ }^{3}$ If first-line treatment becomes ineffective, second-line treatment including rituximab as a B-cell depleting agent and cyclophosphamide as an anti-inflammatory agent would be considered. ${ }^{2}$

As clinicians, it is important to recognise classic features that alert us to the possibility of autoimmune encephalitis. These features include subacute onset of confusion, short-term memory loss, behaviour change (depression, apathy, irritability), and seizures (usual temporal complex partial type). ${ }^{4}$ These features can precede the development of cancer for paraneoplastic encephalitis..$^{5}$ It is important to obtain the history of smoking and family history of malignancy. The occurrence of refractory seizure despite prescription of multiple antiepileptics should prompt the clinician to consider this diagnosis as well. As part of the workup to exclude other differential diagnoses, lumbar puncture, electroencephalogram, and brain MRI should be performed, although these are non-specific tests for confirming autoimmune encephalitis. ${ }^{4}$ A CSF picture of lymphocytosis with mildly elevated protein and presence of oligoclonal bands will be present. ${ }^{4}$ Electroencephalography will show temporal lobe abnormalities, while brain MRI will reveal a unilateral or bilateral hyperintense medial temporal lobe signal change on T2 and fluidattenuated inversion recovery without contrast enhancement that can progress to hippocampal and temporal lobe atrophy. ${ }^{4,6}$

A condition that mimics autoimmune encephalitis is infectious encephalitis, particularly herpes encephalitis. To differentiate the two, several clinical clues might be useful. Fever is almost always present in infectious encephalitis; it is present in about $50 \%$ of autoimmune encephalitis cases. ${ }^{6}$ Skin lesions can be found in varicella-zoster infection. ${ }^{6}$ 
Lymphocytic pleocytosis is milder in autoimmune encephalitis than in viral illnesses. ${ }^{6}$ Periodic lateral epileptic discharge over the temporal region can be found in herpes encephalitis, and typical brain MRI findings in this aetiology would be asymmetric medial temporal lobe necrosis along with cingulate and insular region involvement. ${ }^{6}$ Herpes simplex virus-polymerase chain reaction in CSF would be essential as part of the workup as well. Ultimately, for definitive diagnosis of autoimmune encephalitis, paired serum and CSF antibodies should be obtained. In $\mathrm{GABA}_{\mathrm{B}}$ encephalitis, antibodies target both GABA $_{B 1}$ and ${ }_{B 2}$ subunits located mainly in the hippocampus, thalamus, and cerebellum. ${ }^{7}$ Patients usually have early prominent seizures (temporal lobe epilepsy), memory deficits, increased anxiety, and mood dysregulation. Novel symptoms such as ataxia or opsoclonus-myoclonus have also been recently reported. ${ }^{7,8}$ Small-cell lung carcinoma is often present. ${ }^{7}$ Concurrent antibodies such as VGKC-Ab, GAD-Ab (glutamic acid decarboxylase) have also been documented., ${ }^{7,9}$ The outcome is driven by the adequacy of tumour removal if found and the presence of other auto-antibodies, particularly onconeural antibodies (amphiphysin and SOX1) that are associated with a poorer prognosis. ${ }^{8}$

In a recent literature review, the discovery of anti-GABA antibody as a target of autoimmunity has received much attention as a cause of refractory seizures or status epilepticus. ${ }^{10}$ In contrast to GABA $_{B}$ encephalitis, extensive cortical-subcortical brain MRI abnormalities and the co-existence with other antibodies particularly GAD65 or TPO (thyroid peroxidase) were shown in a recent case series report. ${ }^{10}$

To monitor disease activity, both serum and CSF antibodies should be collected as some are more readily detectable in one compartment than another: NMDA antibodies are readily obtained from CSF and VGKC from serum. ${ }^{2}$ In general, treatment such as long-term immunosuppression should be guided by clinical judgement and not necessarily on antibody level.

In our patient, after a 5-day course of IVIG there was no recurrence of seizure. Oral prednisolone ( $1 \mathrm{mg} / \mathrm{kg} /$ day) was then prescribed and slowly tapered. His cognition was assessed 1 week after IVIG. Although MMSE remained low with a score of $15 / 30$, improvement in abstract thinking, calculation, and proverb interpretation was noted upon discharge.
Positron emission tomography/CT was performed in late March 2014 to screen for malignancy. There was abnormal fluorodeoxyglucose uptake over the medial aspect of the left temporal lobe. Brain MRI was repeated 2 months after discharge and showed resolved signal/swelling in the medial aspect of the left temporal lobe. His cognition was again assessed in November 2014 and showed improvement in orientation and judgement, but still moderately to severely impaired memory.

\section{Declaration}

No conflict of interests was declared by the authors.

\section{Acknowledgement}

I would like to thank Dr Josep Dalmau, Service of Neurology, Hospital Clinic, University of Barcelona, Spain, for his assistance in processing our sample.

\section{References}

1. Didelot A, Honnorat J. Autoimmune limbic encephalitis. Future Neurol 2011;6:97-111.

2. Vincent A, Bien CG, Irani SR, Waters P. Autoantibodies associated with diseases of the CNS: new developments and future challenges. Lancet Neurol 2011;10:759-72.

3. Titulaer MJ, McCracken L, Gabilondo I, et al. Treatment and prognostic factors for long-term outcome in patients with anti-NMDA receptor encephalitis: an observational cohort study. Lancet Neurol 2013;12:157-65.

4. Derry CP, Wilkie MD, Al-Shahi Salman R, Davenport RJ. Autoimmune limbic encephalitis. Clin Med (Lond) 2011;11:476-8

5. Dalmau J, Rosenfeld MR. Paraneoplastic syndromes of the CNS. Lancet Neurol 2008;7:327-40.

6. Armangue T, Leypoldt F, Dalmau J. Autoimmune encephalitis as differential diagnosis of infectious encephalitis. Curr Opin Neurol 2014;27:361-8.

7. Lancaster E, Lai $M$, Peng $X$, et al. Antibodies to the $\mathrm{GABA}_{\mathrm{B}}$ receptor in limbic encephalitis with seizures: case series and characterisation of the antigen. Lancet Neurol 2010;9:67-76.

8. Höftberger R, Titulaer MJ, Sabater L, et al. Encephalitis and $\mathrm{GABA}_{\mathrm{B}}$ receptor antibodies: novel findings in a new case series of 20 patients. Neurology 2013;81:1500-6.

9. Boronat A, Sabater L, Saiz A, Dalmau J, Graus F. GABA receptor antibodies in limbic encephalitis and anti-GADassociated neurologic disorders. Neurology 2011;76:795800.

10. Petit-Pedrol M, Armangue T, Peng X, et al. Encephalitis with refractory seizures, status epilepticus, and antibodies to the $\mathrm{GABA}_{\mathrm{A}}$ receptor: a case series, characterisation of the antigen, and analysis of the effects of antibodies. Lancet Neurol 2014;13:276-86. 